\title{
Structural Characterization of Neutral Oligosaccharides by Laser-Enhanced In-Source Decay of MALDI-FTICR MS
}

\author{
Hongmei Yang, ${ }^{1,2}$ Yingning Yu, ${ }^{1}$ Fengrui Song, ${ }^{1}$ Shuying Liu ${ }^{1,3}$ \\ ${ }^{1}$ Changchun Institute of Applied Chemistry, Chinese Academy of Sciences, 5625 Renmin Street, Changchun 130022, China \\ ${ }^{2}$ Graduate School of the Chinese Academy of Sciences, Beijing 100039, China \\ ${ }^{3}$ Changchun University of Chinese Medicine, 1035 Boshuo Road, Changchun 130117, China
}

\begin{abstract}
MALDI in-source decay (ISD) technique described to date has proven to be a convenient and rapid method for sequencing purified peptides and proteins. However, the general ISD still can not produce adequate fragments for the detailed structural elucidation of oligosaccharides. In this study, an efficient and practical method termed the laser-enhanced ISD (LEISD) technique of MALDI-FTICR MS allows highly reliable and abundant fragmentation of the neutral oligosaccharides, which was attributed to the ultrahigh irradiation laser of $\mathrm{mJ}$ level. The yield of ISD fragmentation was evaluated under different laser powers for 7 neutral oligosaccharides using DHB as matrix. Better quality ISD spectra including fragment ions in low-mass region were obtained at higher laser power. Results from the LEISD of oligosaccharides demonstrated that a significantly better signal-to-noise ratio $(\mathrm{S} / \mathrm{N})$ and more structural information could be obtained in comparison to the conventional CID. It was also suggested that the valuable A ions derived from cross-ring cleavage of the linear oligosaccharides allowed the distinction among $\alpha(1 \rightarrow 4)$-, $\alpha(1 \rightarrow 6)-, \beta(1 \rightarrow 4)$-, and $\beta(1 \rightarrow 3)$-linked isobaric structures according to fragment types and intensities. In addition, ideal fragmentation ions observed by LEISD method facilitated the determination of the sequences and branched points of complex oligosaccharides from human milk.
\end{abstract}

Key words: LEISD technique of MALDI-FTICR MS, Highly reliable and abundant fragmentation, Neutral oligosaccharides, Laser power, Sequences and branched points

\section{Introduction}

$\mathrm{C}$ arbohydrates play important roles in multiple biological processes such as anticoagulants, immunomodulators, antigenic microbial recognition factors, inflammatory response addressins, fertilization, and cell adhesion

Electronic supplementary material The online version of this article (doi:10.1007/s13361-011-0085-0) contains supplementary material, which is available to authorized users.

Correspondence to: Shuying Liu; e-mail: syliu19@yahoo.com.cn molecule, as forms of either glycolipids or glycoproteins [1-3]. Because of the continuing discoveries of new roles for oligosaccharides whose structures are in many cases related to their biological functions, it is important to elucidate their structures. Since the amount of individual carbohydrate species is often severely limited, a sensitive and effective analytical method for oligosaccharides is required. But it is still a greater challenging task given to biochemists.

The conventional analysis for carbohydrates involves a combination of techniques such as NMR, GC/MS, and monosaccharide composition analysis $[4,5]$. With the development of matrix-assisted laser desorption/ionization 
(MALDI) and electrospray ionization (ESI) mass spectrometry (MS), MS-based technology has become a key methodology for structural elucidation of carbohydrates [6, 7]. Cross-ring cleavage ions were found to be dominant for cationized species by using infrared laser desorption and fast atom bombardment MS during early work [8,9]. However, poor signal-to-noise ratio $(\mathrm{S} / \mathrm{N})$ and low sensitivity were found. Several groups have reported the determination of carbohydrates using MALDI time of flight (TOF) MS [1012], which allowed the production of ions from underivatized carbohydrates and a lower detection limit. The structures of oligosaccharides were determined by MALDI post-source decay MS, MALDI-TOF-TOF MS, and infrared multiphoton dissociation (IRMPD) in Fourier transform ion cyclotron resonance (FTICR) MS [13-15]. PSD spectra are often of relatively poor quality from carbohydrates, and information in the lower mass regions of the spectra is difficult to obtain. IRMPD can yield abundant ions for many types of oligosaccharides and provides better S/N. Sequence and linkage information of oligosaccharides can be obtained by these methods.

There are several previous publications on the analysis of carbohydrates involving MALDI-ISD method [16-21]. Harvey et al. observed weak fragment ions in the ISD spectra of N-linked oligosaccharides with a magnetic sector instrument operated at full-power laser $(180 \mu \mathrm{J} /$ pulse $)$ [16]. They also found that the absolute abundances of both glycosidic and cross-ring cleavage products increased with the delay times $(0.65-2.5 \mu \mathrm{s})$ [17]. Interestingly, Lebrilla's group reported a systematic study on the relationship between the fragment ion yields of neutral oligosaccharides and the alkali metals by MALDI FT MS [18]. It was found that the amount of fragments decreased with increasing alkali metal ion sizes. In addition, cross-ring cleavage produced by ISD at the reducing terminal of the oligosaccharides occurred in negative-ion UV-MALDI MS [20]. Relatively limited structural information can be obtained in their reports due to a small quantity of fragmentation ions. In summary, the in-source fragmentation ions were of less than $5 \%$ relative abundance in most positive-ion spectra, and fragment ions in the region below $\mathrm{m} / \mathrm{z} 500$ were difficult to resolve from the abundant matrix ions. Therefore, it is important to note that rich structural information can not be obtained in all the above reports.

In this paper, high numbers of fragmentation ions including cross-ring cleavages in the laser-enhanced ISD (LEISD) spectra of oligosaccharides, acquired on the MALDI-FTICR mass spectrometer equipped with a highenergy laser (50 $\mathrm{mJ}$ per pulse), were observed for the first time, which were attributed to the high irradiation laser. The extent of fragmentation of oligosaccharides is dependent on and can be controlled by adjusting the laser power. The LEISD and collision-induced dissociation (CID) behaviors of oligosaccharides were also compared. Similar fragments for the oligosaccharides were found in LEISD and CID, but LEISD provided a significantly better $\mathrm{S} / \mathrm{N}$ and more structural information. We present data to demonstrate the powerful applicability of the LEISD approach for analysis of neutral oligosaccharides including linkage analysis of structural isomers.

\section{Experimental}

\section{Reagents}

Maltoheptaose was bought from Tokyo Chemical Industry Co., Ltd (Tokyo, Japan). Dextran 1000, difucosyl-paralacto-N-hexaose (DFpLNH II), and difucosyllacto-N-neohexaose (DFLNnH) were purchased from Fluka (Buchs, Switzerland). Inulin, verbascose, raffinose, and 2,5-dihydroxybenzoic acid (DHB) were purchased from Sigma (St. Louis, MO, USA). Laminarihexaose and mannohexaose were acquired from Megazyme International Ireland Ltd. (Bray Business Park, Bray, Co. Wicklow, Ireland). $\alpha$ Cyclodextrin $(\alpha-C D)$, cyclomaltoheptaose ( $\beta$-cyclodextrin, $\beta$-CD), and $\gamma$-cyclodextrin $(\gamma-\mathrm{CD})$ were purchased from Beijing AoBoXing Bio-Technology Co., Ltd. (Beijing, China). Acetonitrile (HPLC grade) was obtained from Fisher Chemical Company. All reagents were purchased in the highest purity and used without further purification. Ultrapure water (specific conductivity, $18.2 \mathrm{M} \Omega / \mathrm{cm}$ ) was produced by a MilliQ device (Millipore, Milford, MA, USA).

\section{Sample Preparation}

Samples for MALDI were prepared on the stainless steel target by mixing $0.5 \mu \mathrm{L}$ of aqueous analyte solution $(0.1 \mu \mathrm{g} / \mu \mathrm{L})$ with $0.5 \mu \mathrm{L}$ of DHB matrix solution. The matrix solution in $50 \%$ water/acetonitrile $(10 \mu \mathrm{g} / \mu \mathrm{L})$ was freshly prepared immediately prior to use. The mixture was dried at ambient temperature prior to MALDI analysis.

\section{MALDI-FTICR-MS}

MALDI mass spectra were acquired on a MALDI-FTICR mass spectrometer (Varian IonSpec, Lake Forest, CA, USA) equipped with a $7.0 \mathrm{~T}$ superconducting magnet and an external MALDI source. An Orion Nd:YAG frequency tripled-laser $(355 \mathrm{~nm}, 50 \mathrm{~mJ} / 5-\mathrm{ns}$ pulse, $1 \mathrm{~Hz})$ promoted the desorption and ionization of the analytes (New Wave Research, Fremont, CA, USA). A laser spot size of $\sim 100 \mu \mathrm{m}$ in diameter was used in this study. The ions were excited prior to detection with a radiofrequency (rf) sweep beginning at $7000 \mathrm{~ms}$ and amplitude of $120 \mathrm{~V}$ (base to peak) for $4 \mathrm{~ms}$. The two trapping plates were initialized to $20 \mathrm{~V}$, and both were ramped to $0 \mathrm{~V}$ from 6000 to $7000 \mathrm{~ms}$ to reduce baseline distortion of peaks. Before each test, the mass spectrometer was calibrated using singly charged monoisotopic peaks (sodium adducts) of an oligosaccharide mixture of raffinose $(527.15826 \mathrm{Da})$, verbascose $(851.26390 \mathrm{Da})$, 
and $\beta$-CD (1157.35899 Da). All mass spectra were obtained by one laser shot corresponding to a single spectrum.

\section{Collision Induced Dissociation}

The sustained off-resonance irradiation CID was performed by isolating the desired ion in the ICR cell using an arbitrary waveform generator. The ions were excited at $800 \mathrm{~Hz}$ offset from their cyclotron frequency for $1000 \mathrm{~ms}$ at $6 \mathrm{Vbp}$. Two nitrogen pulses ( 250 and $400 \mathrm{~ms}$ ) were used during the CID event to maintain a pressure of $10^{-5}$ Torr.

\section{Results and Discussion}

To obtain repeatable and high quality ISD mass spectra of oligosaccharides in this study, the experiments were performed at $90 \%$ full power, unless mentioned otherwise. As a matter of fact, we examined the influence of some commonly used matrices on ISD behavior of oligosaccharides (data not shown), and the most suitable matrix for obtaining rich structural information was DHB. For this reason, the data reported in this study were obtained by using DHB as matrix. The Domon and Costello nomenclature [22] has been employed throughout this work to define the fragment ions from the oligosaccharides. According to this nomenclature, the ions retaining the charge at the nonreducing terminus are designated as $A_{i}$ for cross-ring cleavages, and $B_{i}$ and $C_{i}$ for glycosidic bond cleavages. The subscript $\mathrm{i}$ represents the number of the glycosidic bond cleavage, counted from the non-reducing end. Those retaining the charge at the reducing terminus are designated as $X_{j}$ for cross-ring cleavages, and $Y_{j}$ and $Z_{j}$ for glycosidic bond cleavages. The subscript $\mathrm{j}$ represents the number of the glycosidic bond cleavage, counted from the reducing end. Greek letters are used to distinguish fragments from branched-chain oligosaccharides, with $\alpha$ and $\beta$ representing the largest branch and the branch with decreasing molecular weight, respectively. In the case of ring cleavages, superscript numbers are given to show the ruptured bonds. Oligosaccharides predominately produced sodium-adducted peaks in the positive ion mode.

\section{Influence of the Laser Power on ISD of Oligosaccharides}

The results from LEISD of oligosaccharides obtained using laser of $\mathrm{mJ}$ level in this study were much distinct from those from traditional ISD reported [16-21]. Abundant fragmentation ions were obtained in this work, where laser power played an essential role. The bar graphs of seven analytes in Figure 1 depict the correlation between fragmentation yield and laser power. The intact molecular ions of neutral oligosaccharides as the base peak with little or no fragmentation were observed at $50 \%$ full power because fragment ions appeared above a certain threshold. It is clearly seen that the fragmentation increases dramatically from $50 \%$ to

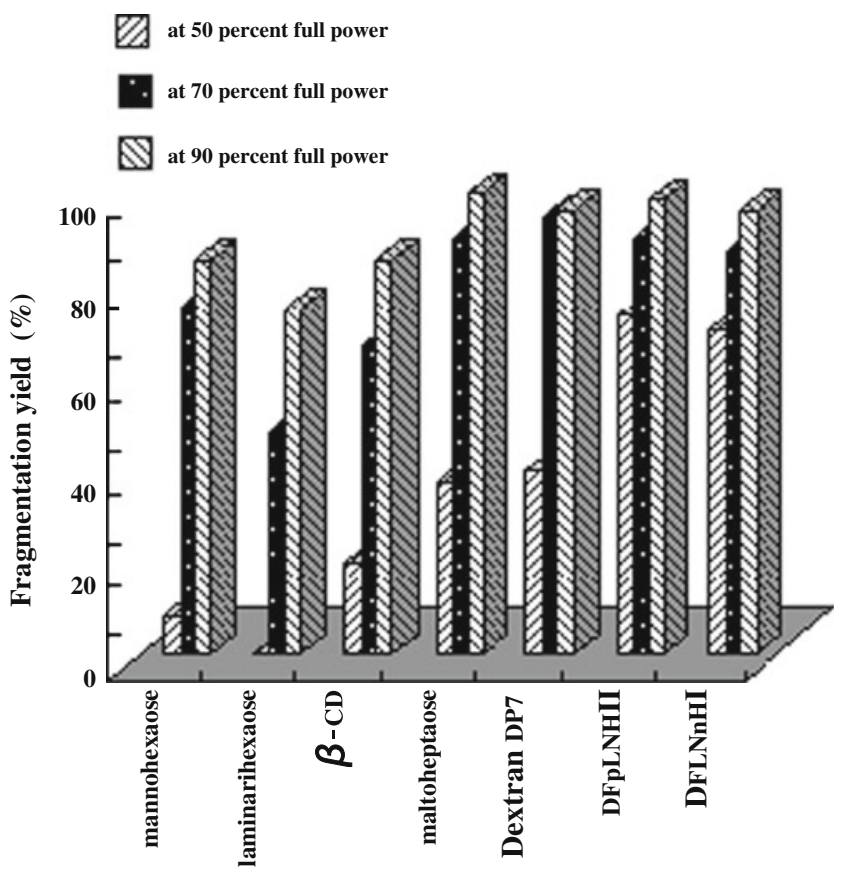

Figure 1. Relation between fragmentation yield and laser power (under identical experimental conditions). Fragmentation yield is calculated as the ratio of the sum of all fragment intensities to the total ion intensity in the ISD mass spectrum of each oligosaccharide

$70 \%$ full power for all the oligosaccharides studied. After passing through about $70 \%$ full power, the fragmentation yields of the analytes with the exception of laminarihexaose and $\beta-C D$ rise slightly with the increased laser power. In comparison with spectra of analytes at $90 \%$ full power, those at the higher laser irradiation ( $>90 \%$ full power) had nominal change (data not shown). Comparison of the fragmentation yields for the seve analytes (see Figures 2, 3, S-1a, and S-2, as well as Scheme 1 for structures) with different laser powers shows that the other relationship, i.e., between oligosaccharide structure and fragmentation yield, is maintained. At the same laser power, the fragmentation yields of different oligosaccharides are as follows: human milk oligosaccharides (DFpLNH II and DFLNnH) $>\alpha$ anomers $>\beta$ anomers, which is related to the stability of the structures. At $50 \%$ full power, the sum of all other fragments together accounts for more than $70 \%$ to the total ion for DFpLNH II and DFLNnH. Our data support the previous report where the oligosaccharides containing fucoses were particularly labile, and losing fucosyl residue required lower energy [23, 24].

Fortunately, increasing the laser power resulted in more fragment ions and simultaneously decreased matrix ions. In order to test this, mannohexaose is taken as an example (Figure S-1a and S-1b). We found that laser power played a very important role in obtaining fragmentations. Seventy percent full power corresponds to an irradiance of about $7 \times$ $10^{9} \mathrm{~W} \cdot \mathrm{cm}^{-2}$ according to the laser spot size and the laser power in this report. For a laser generating pulse energy of 

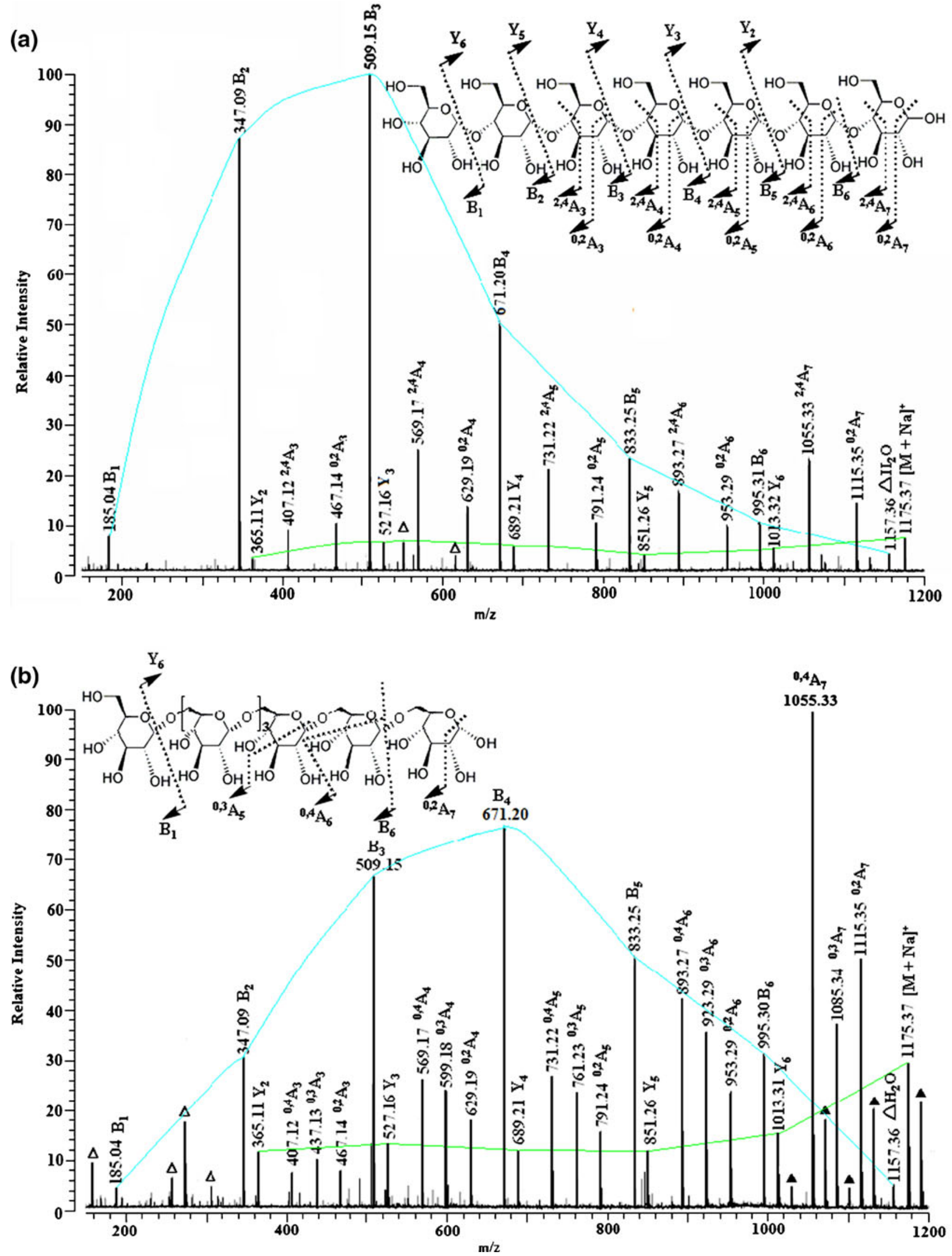

Figure 2. LEISD mass spectra of (a) maltoheptaose $(\alpha-1,4)$ and (b) dextran DP7 ( $(\alpha-1,6)$. DP indicates degree of polymerization. Peaks labeled with hollow triangles are from the matrix. Peaks labeled with filled triangles are potassium adduct ions 


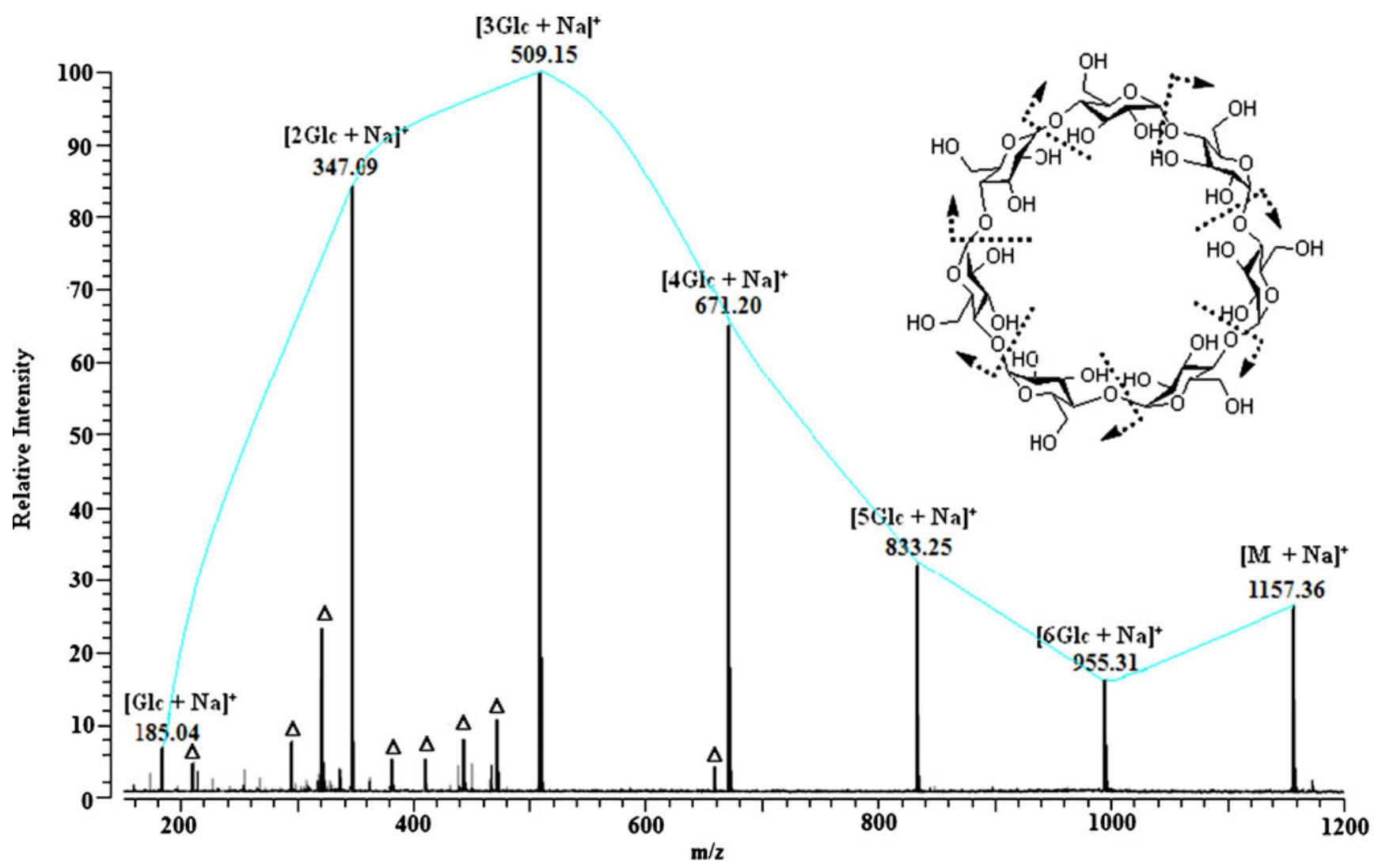

Figure 3. LEISD mass spectrum of $\beta-C D$. Annotation is performed similar to Figure 2

$\mu \mathrm{J}$ levels, the maximum energy flux density is about $10^{7}$ $\mathrm{W} \cdot \mathrm{cm}^{-2}[25]$. Therefore abundant regular cleavage ions were not produced in the mass spectrometer equipped with a common used laser for MALDI MS. Because there are substantial numbers of electrons, protons, and cations in a MALDI plume [26], the origin of ISD is thought to be very complicated. Until now, no uniform mechanism has been proposed to explain ISD processes. The high fragmentation yield in the present LEISD spectra may be due to the induction by hydrogen radicals generated by a photochemical reaction, in-plume thermal activation, or/and collisional activation in the expanding MALDI plume [27-29]. This observation is promised to be helpful for the elucidation of the poorly understood mechanisms of MALDI ISD in our future investigation.

\section{Linear Oligosaccharides with Different Linkages}

The LEISD technique can efficiently distinguish the linkages of linear oligosaccharides. Under the same measurement conditions, both the patterns of ISD ions and their relative ion abundances depend on the oligosaccharide linkage types. The ISD spectrum of $\alpha(1 \rightarrow 4)$ linked maltoheptaose, which is a linear glucose heptamer, illustrated in Figure 2a. The major peaks correspond to Btype ions (i.e., $m / z$ 995.31, 833.25, 671.20, 509.15,
$347.09,185.04)$ in Figure 2a. Besides these ions, crossring cleavage ions at $m / z 1115.35,1055.33$, 953.29, 893.27, 791.24, 731.22, 629.19, 569.17, and 467.14 as well as much less abundant Y-type ions at $\mathrm{m} / \mathrm{z}$ 1013.32, $851.26,689.21,527.16,365.11$ were detected. Figure $2 \mathrm{a}$ clearly indicates the presence of the ${ }^{0,2} \mathrm{~A}$-ion series (i.e., $\mathrm{m} / \mathrm{z} 1115.35,953.29,791.24,629.19,467.14)$ and the ${ }^{2,4}$ A-ion series (i.e., $m / z$ 1055.33, 893.27, 731.22, 569.17, 407.12), of which the high abundances are typical for this spectrum.

The ISD spectrum of the $\alpha(1 \rightarrow 6)$-linked Dextran DP7 (from the LC fraction, separation procedure given in Supplemental Information) is depicted in Figure $2 b$. Compared with the spectrum of maltoheptaose (Figure 2a), the abundant B-ion series whose intensities also constituted a similar envelope, the ${ }^{0,2} \mathrm{~A}$-ion series, and $\mathrm{Y}$-ion series were also observed in that of Dextran DP7. In addition, ions at $m / z 1055.33,893.27,731.22$, 569.17 , and 407.12 in the ISD spectrum of Dextran DP7 are assigned as ${ }^{0,4} \mathrm{~A}$ - but not ${ }^{2,4} \mathrm{~A}$-ion series. The ${ }^{0,4} \mathrm{~A}$-ion series derived from Dextran DP7 are more intense than the ${ }^{2,4} \mathrm{~A}$-ion series of maltoheptaose, which indicates requirement of higher energy for the formation of ${ }^{2,4} \mathrm{~A}$ ion series from the latter.

The difference between the spectra of $\alpha(1 \rightarrow 4)$ - and $\alpha(1 \rightarrow 6)$-linked oligosaccharides, besides the intensity of A- 

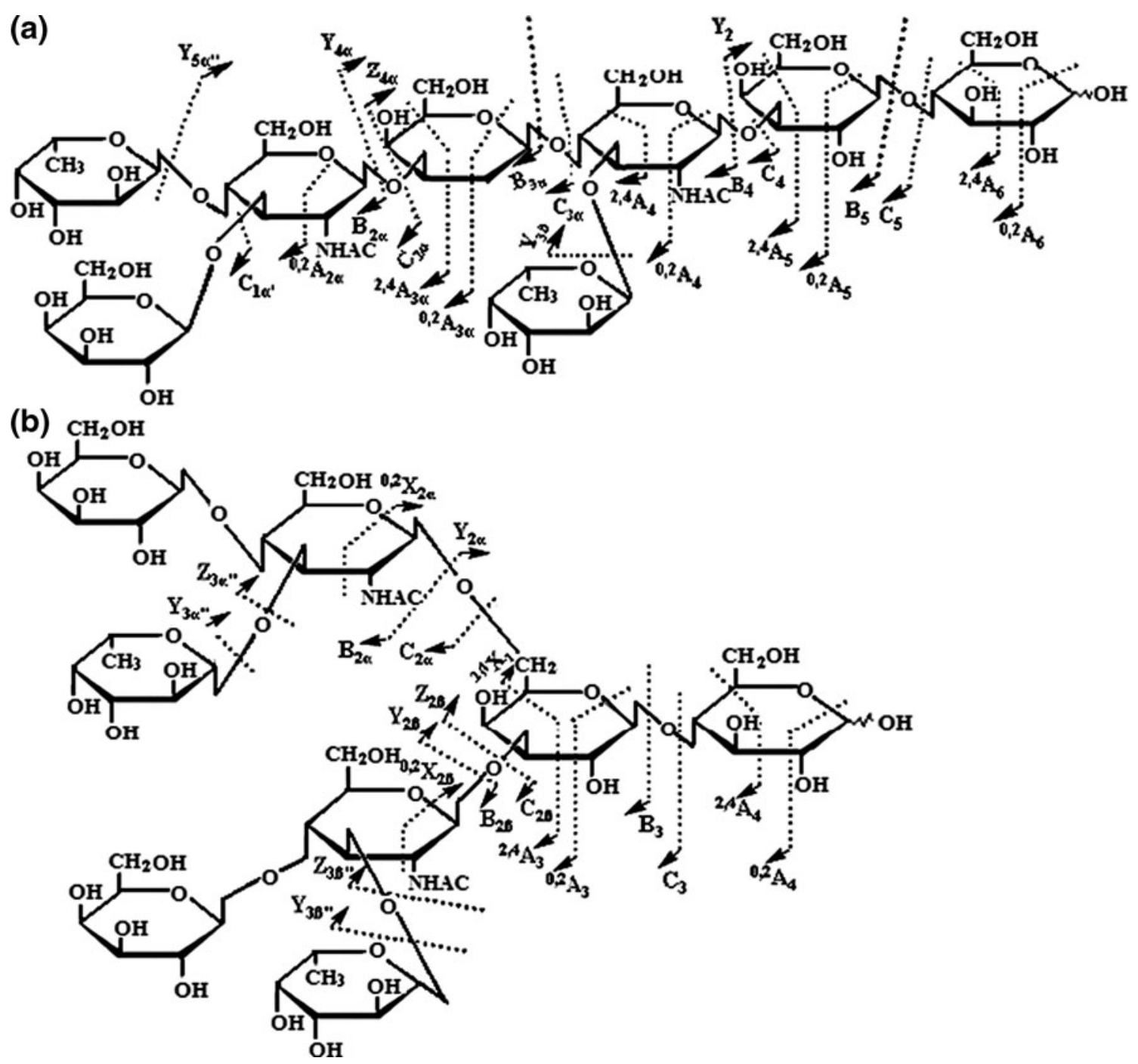

Scheme 1. Glycosidic and cross-ring cleavages found in the LEISD spectra of (a) DFpLNH II and (b) DFLNnH

ion series, is also observed in the ions (i.e., $\mathrm{m} / \mathrm{z} 1085.34$, 923.29, 761.23, 599.18, and 437.13) from ${ }^{0,3} \mathrm{~A}$ cross-ring fragmentations; i.e., ${ }^{0,3} \mathrm{~A}$-ion series can only be observed in the spectrum of the $\alpha(1 \rightarrow 6)$-linked oligosaccharide (Figure $2 b$ ), but not in that of the $\alpha(1 \rightarrow 4)$-linked one. The results showed that the oligosaccharide containing multiple $\alpha(1 \rightarrow 6)$ glycosidic bonds was cleaved more easily during ISD at the pyranose ring than the oligosaccharide with multiple $\alpha(1 \rightarrow 4)$ bonds, which is known to form a helical coil. Unlike the report [14] where the two isobaric structures were distinguished by the fine distinction of the A-type fragments, isomers between the $\alpha(1 \rightarrow 4)$ - and $\alpha(1 \rightarrow 6)$ - linked oligosaccharides were easily distinguished just by identifying the presence of ${ }^{0,3} \mathrm{~A}$-ion series in this work.

In addition, $\beta(1 \rightarrow 4)$-linked mannohexaose and $\beta(1 \rightarrow$ 3)-linked laminarihexaose are selected as model compounds to study the fragmentation characteristics of oligosaccharides in $\beta$-anomeric configuration by LEISD. Figures S-1 and S-2 of Supplemental Information describe the LEISD spectra for mannohexaose and laminarihexaose, respectively, and their detailed elucidation is described in Supplemental Information. It is easy to differentiate among $\alpha(1 \rightarrow 4)-, \alpha(1 \rightarrow 6)-, \beta(1 \rightarrow 4)-$, and $\beta(1 \rightarrow 3)$-linked structures, as listed in Table 1 .

Table 1. Fragmentation characteristics of linear oligosaccharides by the LEISD approach

\begin{tabular}{llcr}
\hline Linkage & \multicolumn{1}{c}{ A-Type ions } & B:Y Ratio & Relative Ion Abundance \\
\hline$\alpha(1 \rightarrow 4)$ & ${ }^{0,2} \mathrm{~A}(-60),{ }^{2,4} \mathrm{~A}(-120)$ & $>>1$ & ${ }^{2,4} \mathrm{~A}>^{0,2} \mathrm{~A}$ \\
$\alpha(1 \rightarrow 6)$ & ${ }^{0,2} \mathrm{~A}(-60),{ }^{0,3} \mathrm{~A}(-90),{ }^{0,4} \mathrm{~A}(-20)$ & $>>1$ & $0,4>^{0,3} \mathrm{~A}>^{0,2} \mathrm{~A}$ \\
$\beta(1 \rightarrow 4)$ & ${ }^{0,2} \mathrm{~A}(-60),{ }^{2,4} \mathrm{~A}(-120)$ & $>1$ & ${ }^{0,2} \mathrm{~A}>^{2,4} \mathrm{~A}$ \\
$\beta(1 \rightarrow 3)$ & $0,2 \mathrm{~A}(-60),{ }^{1,4} \mathrm{~A}(-90),{ }^{2,4} \mathrm{~A}(-120)$ & $\approx 1$ & $1,4 \mathrm{~A}>>^{0,2} \mathrm{~A} \approx^{2,4} \mathrm{~A}$ \\
\hline
\end{tabular}


Abundant cross-ring fragmentations, which are only commonly observed by high-energy collision-induced dissociation (heCID) technology for analysis of native oligosaccharides [30-32], were observed in the ISD spectra. The fragmentations could be dynamically controlled; therefore, the intensities of ions could be described by Arrhenius equation, in which the front exponential factor A reflects the structural characteristics. There are some striking advantages of the LEISD method over the previously reported ISD methods [16-21], such as better $\mathrm{S} / \mathrm{N}$, more extensive fragmentation, and no interference from matrix ions. Better results obtained in positive-ion mode with continuous extraction in our experiments were probably because the laser energy $(50 \mathrm{~mJ}$ per pulse) used here was much higher than that used in any previously reported ISD method.

\section{Cyclic Oligosaccharides}

Interestingly, cross-ring cleavage ions were not observed in the spectrum of cyclic oligosaccharides, which allowed easy discrimination between linear oligosaccharides and cyclic ones. The data for $\alpha-C D$ and $\gamma-C D$ are very similar to those for $\beta-C D$ and not provided in this report. The LEISD spectrum of $\beta-C D$ containing seven glucose residues in a ring is indicated in Figure 3, where the differences of the major ions were $162 \mathrm{Da}$ corresponding to one glucose residue. Each fragment peak corresponded to the chemical species of $[6 \mathrm{Glc}+\mathrm{Na}]^{+},[5 \mathrm{Glc}+\mathrm{Na}]^{+},[4 \mathrm{Glc}+\mathrm{Na}]^{+},[3 \mathrm{Glc}+$ $\mathrm{Na}]^{+},[2 \mathrm{Glc}+\mathrm{Na}]^{+}$, and $[\mathrm{Glc}+\mathrm{Na}]^{+}$. The intensity of the fragment ion is highest at $\mathrm{m} / \mathrm{z} 509.15\left([3 \mathrm{Glc}+\mathrm{Na}]^{+}\right)$and the envelope of the intensities of the fragment ions in the spectrum of $\beta-\mathrm{CD}$ (Figure 3) is quite similar to that in the electron impact ionization (EI) mass spectrum of cycloheptane. LEISD approach is collected the merits of MALDI and EI for analysis of underivatized oligosaccharides. Although cleavages were fundamentally unobtainable from ISD in the negative-ion or positive-ion MALDI ionization of cyclodextrins $[19,20]$ the glycosidic bond breakages were detected in our positive MALDI-FTMS spectrum of $\beta$-CD. Comparing Figure 2a with Figure 3, cross-ring cleavage ions were not observed in the spectrum of $\beta-C D$ but were observed as strong signals in that of linear maltoheptaose. Even at very higher laser powers, cross-ring cleavage ions were not observed for $\beta-C D$ owing to its stability. The presence or absence of cross-ring cleavage ions allowed easy discrimination between linear oligosaccharides and cyclic ones, both of which were connected by $\alpha(1 \rightarrow 4)$ glycosidic linkages.

\section{Underivatized Neutral Human Milk Oligosaccharides}

As expected, the new method is also applicable for the analysis of complex oligosaccharides. An example of this application was demonstrated with two difucosylated oligosaccharides, DFpLNH II and DFLNnH (structures given in
Scheme 1). DFpLNH II contains a type 1 (Galß1-3GlcNAc) chain at the nonreducing terminal, while DFLNnH has two type 2 (GalB1-4GlcNAc) chains. The LEISD spectra for DFpLNH II and DFLNnH are shown in Figures 4 and 5, respectively. Fragmentations responsible for the major ions are shown in Scheme 1a and b. Two consecutive fucose residue (146 Da) losses from the intact molecular ions of neutral oligosaccharides, i.e., $m / z 1241.42(\mathrm{M}-146)$ and $1095.37(\mathrm{M}-146 \times 2)$, are observed in Figures 4 and 5, indicating the two molecules are difucosylated. The fragment ions further dissociate to produce strong signals containing only one fucose residue or no fucose residue. In contrast, fragment ions containing two fucose residues, such as ions at $m / z 1225.44$ for DFpLNH II and $1207.43\left(\mathrm{~B}_{3}\right)$ for DFLNnH, are minor and absent, respectively, as shown in Figures 4 and 5.

The major ions were formed by B- and Y-type glycosidic cleavages. Some minor C- and Z-type ions were also observed. It was not possible to determine if a $\mathrm{Z}$ cleavage occurred at the 6-position of the branching Gal for DFLNnH because the resulting fragment ion $(\mathrm{m} / z$ 858.29) was isobaric with the $Z_{2 \beta}$ ion. On the basis of reports where linkage at the 6-position resulted in the absence of the corresponding Z-type ion [31, 32], ions with $m / z 858.29$ and 712.23 in Figure 5 are assigned as $Z_{2 \beta}$ and $Y_{3 \alpha^{\prime \prime}} / Z_{2 \beta}$, respectively. Lots of subsequent losses, which helped to elucidate structures, were observed due to the facile loss of fucose residue, as stated earlier. These ions are the result of several bond cleavages involving losses from both ends of the molecule (termed internal cleavage ions). For clarity, the internal cleavages are designated by " " " and include several series such as $\mathrm{B}_{\mathrm{n}}$ and $\mathrm{Y}_{\mathrm{n}}$. For example, the $\mathrm{B}_{4} / \mathrm{Y}_{5 \alpha^{\prime \prime} / 3 \beta}$ fragment $(\mathrm{m} / \mathrm{z}$ 753.25) in Figure 4 comes from the loss of Gal-Glc at the nonreducing end and two fucose residues. But the genesis of them is not clear in some cases, as several alternate cleavages could produce ions of the same mass. For example, the ion labeled $\mathrm{H}_{2} \mathrm{~N}_{2}(m / z 753.25)$ in Figure 5 could arise from losses of a Glc, two fucose residues, and one of the Gal residues at 3positions of the two GlcNAc residues, or from losses of all the substitutions at 3- and 4-positions of the two GlcNAc residues. The ions of undetermined origin, which result from cleavages of two glycosidic bonds besides the glycosidic bonds linking the fucose residues, are in relatively low abundance as shown in Figures 4 and 5.

One of the most intense ions observed in the spectra of both DFpLNH II and DFLNnH was $m / z$ 388.12. That in Figure 5 termed ion $\mathrm{D}$ is produced by cleavage of one of the antennae $(2 \alpha$ or $2 \beta)$, together with elimination of the substituent linked to $\mathrm{C}-3$ of the GlcNAc residue from the fragment ion $m / z 534.18$ (Gal $\beta 1-4$ (Fuc $\alpha 1-3)$ GlcNAc). This double glycosidic cleavage preferentially occurs at the 3and not the 4-position and is similar to the previously observed D-type fragmentation for the 3-substituted GlcNAc- or Glc-containing oligosaccharides [33-35]. However, the signal observed at $m / z 388.12$ in Figure 4 is not assigned as ion D since it arises from elimination of the 

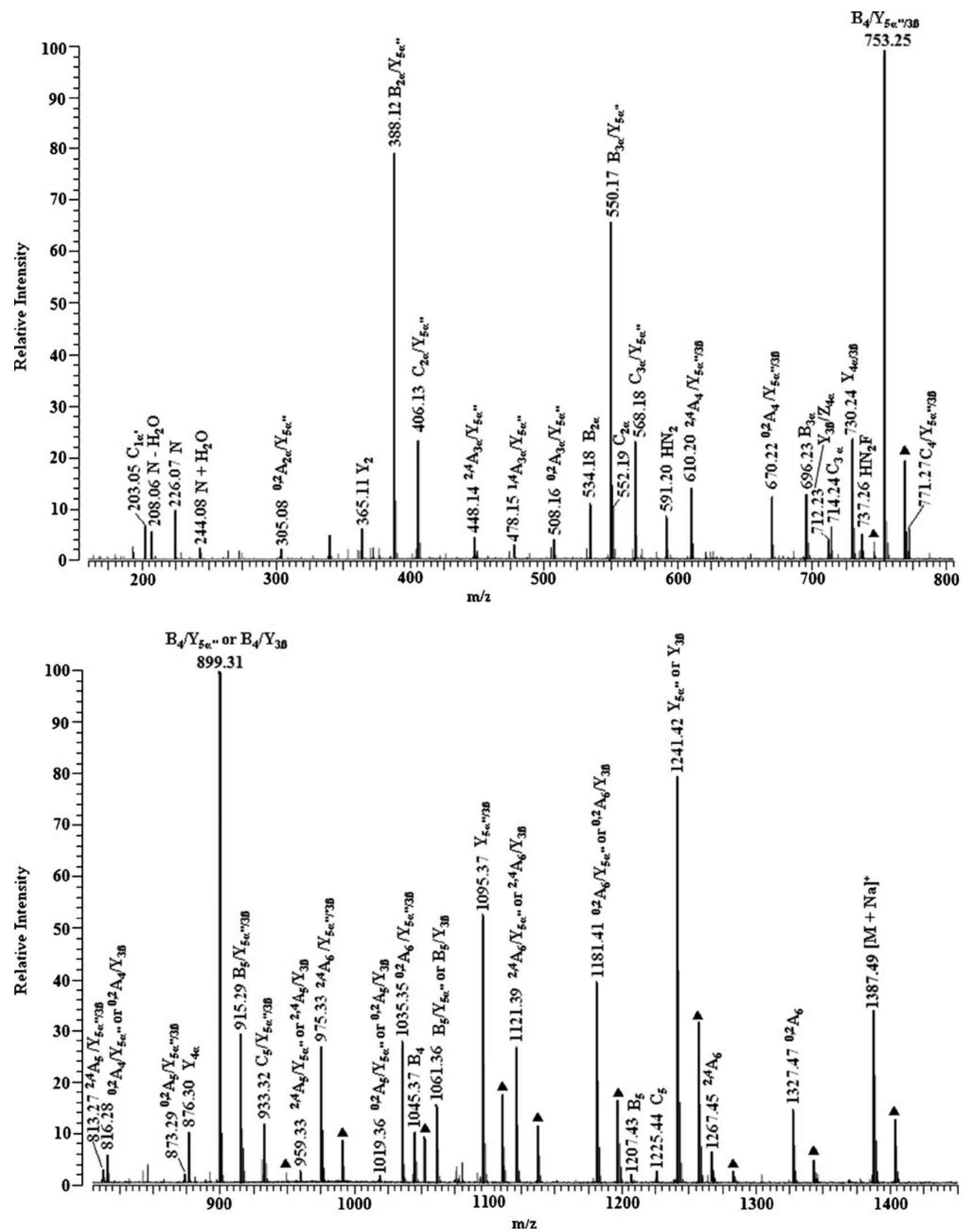

Figure 4. LEISD mass spectrum of DFpLNH II. Peaks labeled with filled triangles are potassium adduct ions. Symbols used to describe the monosaccharide residues are: $\mathrm{F}=$ fucose, $\mathrm{H}=$ hexose, and $\mathrm{N}=\mathrm{GlcNAc}$. The ions labeled $\mathrm{HN}_{2}(\mathrm{~m} / z$ 591.20) and $\mathrm{HN}_{2} \mathrm{~F}(\mathrm{~m} / \mathrm{z} 737.26)$ are internal fragments with composition of (Gal)(GlcNAc) $)_{2}$ and (Gal)(GlcNAc) $)_{2}(\mathrm{Fuc})$, respectively, but of undetermined origin. Signals in the mass range between 805 and 1450 are enhanced by 1.35 times 

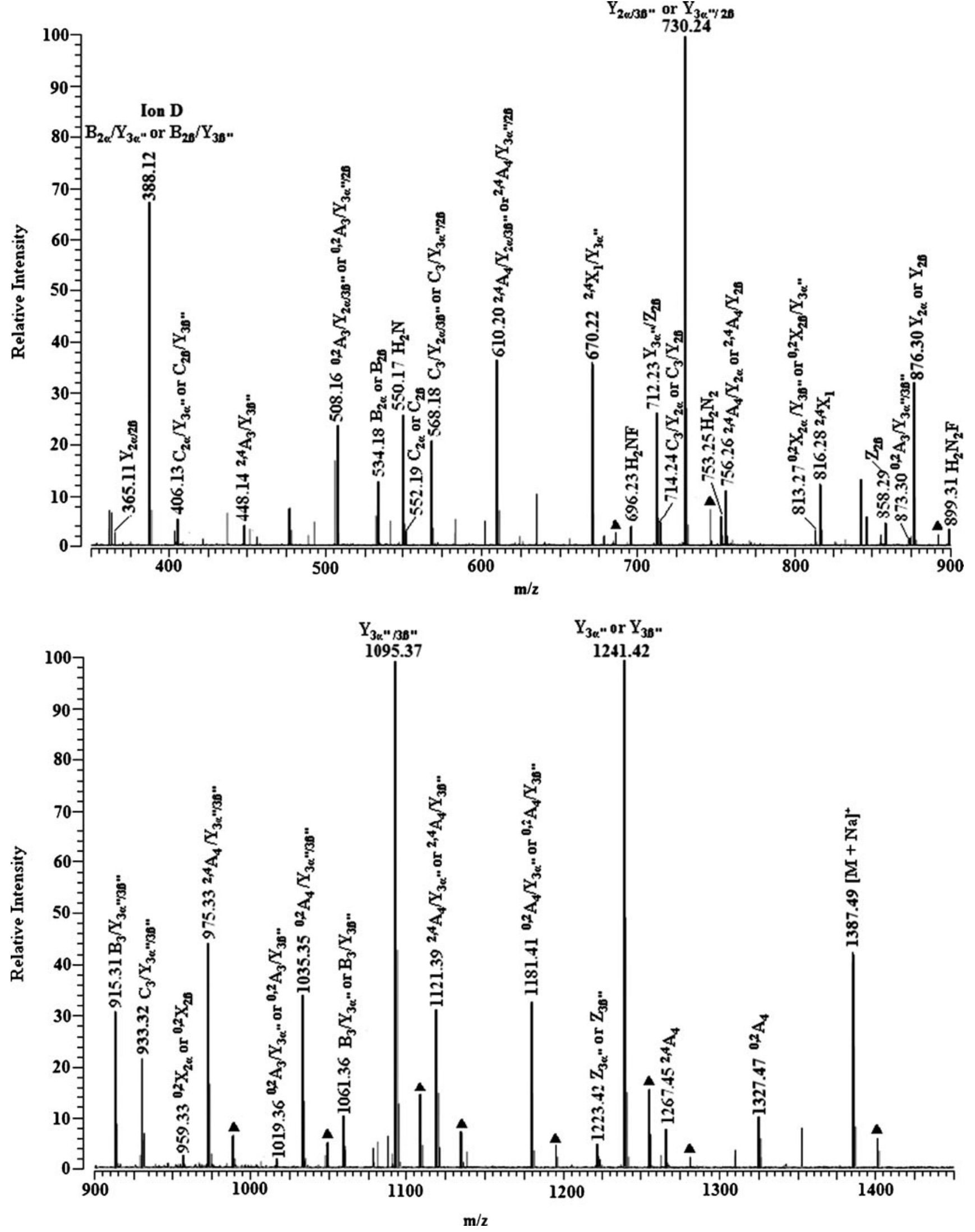

Figure 5. LEISD mass spectrum of DFLNnH. Annotation is performed similar to Figure 4. $\left.\mathrm{H}_{2} \mathrm{~N}(\mathrm{~m} / \mathrm{z} 550.17 \text {, (Hex) })_{2} \mathrm{GlcNAc}\right)$, $\mathrm{H}_{2} \mathrm{NF}(\mathrm{m} / \mathrm{z} \text { 696.23, (Hex) })_{2}$ (GlcNAc)Fuc), $\left.\mathrm{H}_{2} \mathrm{~N}_{2}(\mathrm{~m} / \mathrm{z} \text { 753.25, (Hex) })_{2}(\mathrm{GlcNAc})_{2}\right)$, and $\left.\mathrm{H}_{2} \mathrm{~N}_{2} \mathrm{~F}(\mathrm{~m} / \mathrm{z} \text { 899.31, (Hex) })_{2}(\mathrm{GlcNAc})_{2} \mathrm{Fuc}\right)$ are internal cleavage ions of undetermined origin. Signals in the mass range between 900 and 1450 are enhanced by 1.39 times 
fucose residue linked to $\mathrm{C}-4$ of the GlcNAc residue from the fragment (Gal $\beta 1-3$ (Fuc $\beta 1-4)$ GlcNAc). B $_{2 \alpha}$ ion $(m / z 534.18)$ aided in determination of the presence of Gal $\beta 1-3$ (Fuc $\beta 1-4$ ) GlcNAc in the structure of DFpLNH II. The unique ions at $\mathrm{m} / \mathrm{z} 203.05,208.06,226.07$, and 244.08 (the probable formation routes given in Scheme S-1) are present in the spectrum of DFpLNH II, but not in that of DFLNnH, revealing terminal Gal to be linked to the 3-position of the GlcNAc in DFpLNH II. Hence, the presence or absence of the above four ions serves to differentiate between the type 1 and type 2 chains. In addition, the ion at $\mathrm{m} / z 899.31$ in Figure 4 and the one labeled $\mathrm{H}_{2} \mathrm{NF}(\mathrm{m} / z$ 696.23) in Figure 5 may mainly correspond to D-type ions. The partial sequence of DFpLNH II from the nonreducing end: Hex-(Fuc) HexNAc-Hex-HexNAc-Hex-, can be deduced from the above discussion about the ion at $\mathrm{m} / \mathrm{z} 388.12$ and the prominent ions at $\mathrm{m} / \mathrm{z} 388.12,550.17(\Delta \mathrm{m}=162 \mathrm{Da}), 753.25$ $(\Delta \mathrm{m}=203 \mathrm{Da})$, and $915.29(\Delta \mathrm{m}=162 \mathrm{Da})$ in Figure 4. The position of another fucose on the third monosaccharide residue (HexNAc) from the reducing end in the structure of DFpLNH II is deduced from the presence of the ion at $\mathrm{m} / \mathrm{z}$ $1045.37(753+146 \times 2)$ and the absence of the ion at $\mathrm{m} / \mathrm{z} 842$ $(550+146 \times 2)$. Its position was also revealed by the mass difference of $349 \mathrm{Da}$ (Fuc+HexNAc) between $\mathrm{B}_{4}(\mathrm{~m} / \mathrm{z}$ 1045.37) and $\mathrm{B}_{3 \alpha}(\mathrm{m} / z$ 696.23). The hexose residue at the reducing end can be inferred from the $162 \mathrm{Da}$ molecular weight difference between DFpLNH II (1364 Da) and the obtained partial sequence (Hex-(Fuc)HexNAc-Hex-(Fuc) HexNAc-Hex, 1202 Da).

A number of cross-ring cleavages throughout the mass range, comprising mainly of ${ }^{0,2} \mathrm{~A}$-ion series and ${ }^{2,4} \mathrm{~A}$-ion series which can provide partial linkage information, were observed for both oligosaccharides. For example, the intensity of ${ }^{0,2} \mathrm{~A}_{6}$ ion $(\mathrm{m} / \mathrm{z} 1327.47)$ is higher than that of ${ }^{2,4} \mathrm{~A}_{6}$ ion $(m / z 1267.45)$ in Figure 4 , which is in line with the ISD characteristic of $\beta(1 \rightarrow 4)$-linked oligosaccharide, as described in Figure S-1a. Thus, $\beta(1 \rightarrow 4)$-linkage between the two monosaccharide residues at the reducing end can be deduced. Furthermore, the ISD of the human milk oligosaccharides yielded a number of internal cross-ring cleavages. These cleavages such as ${ }^{2,4} \mathrm{~A}_{4} / \mathrm{Y}_{5 \alpha^{\prime \prime} / 3 \beta}(\mathrm{m} / z$ 610.20) in Figure 4 and ${ }^{0,2} \mathrm{~A}_{3} / \mathrm{Y}_{2 \alpha / 3 \beta^{\prime \prime}}(\mathrm{m} / \mathrm{z}$ 508.16) in Figure 5 correspond to the fragmentation at the branched points. They are capable of ruling out some untrue linkages. A few $\mathrm{X}$-type cross-ring cleavage ions are observed in the spectrum of DFLNnH (Figure 5). The ${ }^{2,4} \mathrm{X}_{1}(\mathrm{~m} / \mathrm{z}$ 816.28) served to determine the position of the antenna at C- 6 of the Gal residue. In conclusion, the sequence, partial linkages, and branched points of the human milk oligosaccharides can be obtained by the LEISD method.

It should be mentioned that unique D-type cleavage was not found in any previously reported ISD method. Furthermore, structural information yielded in the present LEISD method is also more than that in the previously reported negative ion ESI tandem MS [34-37]. Types and extent of fragmentation these neutral oligosaccharides experienced in our experiments are similar to those observed earlier in heCID-MALDI-TOF/TOF MS [30-32], where dominant cross-ring fragmentation ions, glycosidic cleavage ions, and internal cleavage ions were observed in the mass spectra of neutral oligosaccharides. The only difference is that ${ }^{1,5} \mathrm{X}$ type cross-ring cleavages in heCID were absent in our study.

\section{ISD and CID Comparison of the Neutral Oligosaccharides}

We also examined commonly used CID mass spectra of all the oligosaccharides studied in order to compare the structural information obtained from ISD and CID. The results showed that the LEISD provided more structural information and a better S/N. Here, we took DFpLNH II as an example. The multistage CID spectra of DFpLNH II are illustrated in Figure S-3. In the $\mathrm{MS}^{2}$ spectrum (Figure S-3a), the ion at $\mathrm{m} / \mathrm{z} 1241.42$ has the highest intensity, belonging to the loss of one fucose residue from the precursor ion. A cross-ring cleavage of the reducing end, ${ }^{0,2} \mathrm{~A}_{6}$, was the largest one among other detected peaks. As shown in the $\mathrm{MS}^{3}$ spectrum (Figure S-3b), subsequent fucose losses from the precursor ion at $\mathrm{m} / z 1241.42$ are clearly discernible (i.e., $\mathrm{m} / \mathrm{z} 1095.37$ and 899.31). A dominant fragment ion $(\mathrm{m} / \mathrm{z}$ 1181.41), which was originated from cross-ring cleavage of the reducing end of the precursor ion, was also observed. However, fragment ions below $m / z 700$ were not observed. Therefore, the CID of this compound did not provide the complete sequence and branched point information even after $\mathrm{MS}^{3}$. By contrast, the LEISD spectrum of DFpLNH II (Figure 4) shows more structural information and a better $\mathrm{S} /$ N. For this instrument with three-stage CID at most, the CID method is not applicable for analysis of labile oligosaccharides.

\section{Conclusions}

The results obtained using the MALDI mass spectrometer equipped with a laser of $\mathrm{mJ}$ level, demonstrate the ability of LEISD to generate desired structural information. The laser power played an important part in obtaining high fragmentation yield and high quality spectra. It is worth noting that both the intact molecular ions of neutral oligosaccharides with fewer or no fragment ions and prominent fragments down to the last residue can be obtained by adjusting the laser power. The LEISD spectra of linear oligosaccharides exhibited abundant cross-ring fragmentation ions and glycosidic cleavages that provide insight into the oligosaccharide linkage and sequence, respectively. The A-type ions observed for the linear oligosaccharides allowed distinction among $\alpha(1 \rightarrow 4)-, \alpha(1 \rightarrow 6)-, \beta(1 \rightarrow 4)$-, and $\beta(1 \rightarrow 3)$-linked structures. Underivatized neutral human milk oligosaccharides were also comprehensively characterized using the LEISD method, and prominent "internal" cleavage ions were observed allowing the determination of branched points. Particularly, the distinctive D-type ion and triplet ions $(\mathrm{m} / \mathrm{z}$ 
208, 226, and 244) can provide partial linkage information and are useful to define oligosaccharide type 1 and type 2 chains. In contrast to CID, LEISD generated better quality spectra with rich structural information. Consequently, this study opens the way to the detailed structural characterization of neutral oligosaccharides by LEISD approach showing rapidity, usefulness, and robustness. There is, therefore, no doubt that the relatively high fragmentation yield of LEISD creates favorable conditions for the future study of the mechanism of MALDI ISD.

\section{Acknowledgment}

The authors acknowledge support for this work by the National Natural Science Foundation of China (no. 20873137). They thank Professor Ning Liu for the technical assistance.

\section{References}

1. Hakomori, S.: The glycosynapse. Proc. Natl. Acad. Sci. U.S.A. 99, 225232 (2002)

2. Dwek, R.A.: Glycobiology: toward understanding the function of sugars. Chem. Rev. 96, 683-720 (1996)

3. Zhang, X.L.: Roles of glycans and glycopeptides in immune system and immune-related diseases. Curr. Med. Chem. 13, 1141-1147 (2006)

4. Bock, K., Schuster-Kolbe, J., Altman, E., Allmaier, G., Stahl, B., Christian, R., Sleytr, U.B., Messner, P.: Primary structure of the oglycosidically linked glycan chain of the crystalline surface layer glycoprotein of Thermoanaerobacter thermohydrosulfuricus L111-69. J. Biol. Chem. 269, 7137-7144 (1994)

5. ZaÈhringer, U., Wagner, F., Rietschel, E.T., Ben-Menachem, G., Deutsch, J., Rottem, S.: Primary structure of a new phosphocholinecontaining glycoglycerolipid of Mycoplasma fermentans. J. Biol. Chem. 272, 3511-3519 (1997)

6. Harvey, D.J.: Analysis of carbohydrates and glycoconjugates by matrixassisted laser desorption/ionization mass spectrometry: an update for the period 2005-2006. Mass Spectrom. Rev. 30, 1-100 (2011)

7. Dell, A., Morris, H.R.: Glycoprotein structure determination by mass spectrometry. Science 291, 2351-2356 (2001)

8. Spengler, B., Dolce, J.W., Cotter, R.J.: Infrared laser desorption mass spectrometry of oligosaccharides: fragmentation mechanisms and isomer analysis. Anal. Chem. 62, 1731-1737 (1990)

9. Orlando, R., Fenselau, C., Cotter, R.J.: Endothermic ion-molecule reactions. 4. Site-directed fragmentation in n-acetylated oligosaccharides at low beam energies. Anal. Chem. 62, 2388-2390 (1990)

10. Zaia, J.: Mass spectrometry of oligosaccharides. Mass Spectrom. Rev. 23, 161-227 (2004)

11. Morelle, W., Faid, V., Chirat, F., Michalski, J.C.: Analysis of N- and OLinked glycans from glycoproteins using MALDI-TOF mass spectrometry. Meth. Mol. Biol. 534, 5-21 (2009)

12. Lastovickova, M., Chmelik, J.: Simple and fast method for recognition of reducing and nonreducing neutral carbohydrates by matrix-assisted laser desorption/ionization time-of-flight mass spectrometry. J. Agric. Food Chem. 54, 5092-5097 (2006)

13. Cai, Y., Jiang, Y., Cole, R.B.: Anionic adducts of oligosaccharides by matrix-assisted laser desorption/ionization time-of-flight mass spectrometry. Anal. Chem. 75, 1638-1644 (2003)

14. Mechref, Y., Novotny, M.V.: Structural characterization of oligosaccharides using MALDI-TOF/TOF tandem mass spectrometry. Anal. Chem. 75, 4895-4903 (2003)

15. Li, B., An, H.J., Hedrick, J.L., Lebrilla, C.B.: Infrared multiphoton dissociation mass spectrometry for structural elucidation of oligosaccharides. Meth. Mol. Biol. 534, 23-35 (2009)

16. Harvey, D.J., Naven, T.J., Küster, B., Bateman, R.H., Green, M.R., Critchley, G.: Comparison of fragmentation modes for the structural determination of complex oligosaccharides ionized by matrix-assisted laser desorption ionization mass spectrometry. Rapid Commun. Mass Spectrom. 9, 1556-1561 (1995)
17. Naven, T.J.P., Harvey, D.J., Brown, J., Critchley, G.: Fragmentation of complex carbohydrates following ionization by matrix-assisted laser desorption with an instrument fitted with time-lag focusing. Rapid Commun. Mass Spectrom. 11, 1681-1686 (1997)

18. Cancilla, M.T., Penn, S.G., Carroll, J.A., Lebrilla, C.B.: Coordination of alkali metals to oligosaccharides dictates fragmentation behavior in matrix assisted laser desorption ionization/fourier fransform mass spectrometry. J. Am. Chem. Soc. 118, 6736-6745 (1996)

19. Bashir, S., Giannakopulos, A.E., Derrick, P.J., Critchley, P., Bottrill, A., Padley, H.D.: Matrix-assisted laser desorption/ionization time-of-flight mass spectrometry. A comparison of fragmentation patterns of linear dextran obtained by in-source decay, post-source decay, and collisioninduced dissociation and the stability of linear and cyclic glucans studied by in-source decay. Eur. J. Mass Spectrom. 10, 109-120 (2004)

20. Yamagaki, T., Suzuki, H., Tachibana, K.: In-source and post-source decay in negative-ion matrix-assisted laser desorption/ionization timeof-flight mass spectrometry of neutral oligosaccharides. Anal. Chem. 77, 1701-1707 (2005)

21. Smargiasso, N., De Pauw, E.: Optimization of matrix conditions for the control of MALDI in-source decay of permethylated glycans. Anal. Chem. 82, 9248-9253 (2010)

22. Domon, B., Costello, C.E.: A systematic nomenclature for carbohydrate fragmentations in FAB-MS/MS spectra of glycoconjugates. Glycoconj. J. 5, 397-409 (1988)

23. Zhang, J., LaMotte, L., Dodds, E.D., Lebrilla, C.B.: Atmospheric pressure MALDI fourier transform mass spectrometry of labile oligosaccharides. Anal. Chem. 77, 4429-4438 (2005)

24. Penn, S.G., Cancilla, M.T., Lebrilla, C.B.: Collision-induced dissociation of branched oligosaccharide ions with analysis and calculation of relative dissociation thresholds. Anal. Chem. 68, 2331-2339 (1996)

25. Dreisewerd, K.: The desorption process in MALDI. Chem. Rev. 103, 395-425 (2003)

26. Knochenmuss, R., Zenobi, R.: MALDI ionization: the role of in-plume processes. Chem. Rev. 103, 441-452 (2003)

27. Köcher, T., Engström, A., Zubarev, R.A.: Fragmentation of peptides in MALDI in-source decay mediated by hydrogen radicals. Anal. Chem. 77, 172-177 (2005)

28. Gabelica, V., Schulz, E., Karas, M.: Internal energy build-up in matrixassisted laser desorption/ionization. J. Mass Spectrom. 39, 579-593 (2004)

29. Luo, G., Marginean, I., Ye, L., Vertes, A.: Competing ion decomposition channels in matrix-assisted laser desorption ionization. J. Phys. Chem. B 112, 6952-6956 (2008)

30. Stephens, E., Maslen, S.L., Green, L.G., Williams, D.H.: Fragmentation characteristics of neutral n-linked glycans using a MALDI-TOF/TOF tandem mass spectrometer. Anal. Chem. 76, 2343-2354 (2004)

31. Lewandrowski, U., Resemann, A., Sickmann, A.: Laser-induced dissociation/high-energy collision-induced dissociation fragmentation using MALDI-TOF/TOF-MS instrumentation for the analysis of neutral and acidic oligosaccharides. Anal. Chem. 77, 3274-3283 (2005)

32. Harvey, D.J., Bateman, R.H., Green, M.R.: High-energy collisioninduced fragmentation of complex oligosaccharides ionized by matrixassisted laser desorption/ionization mass spectrometry. J. Mass Spectrom. 32, 167-187 (1997)

33. Amano, J., Osanai, M., Orita, T., Sugahara, D., Osumi, K.: Structural determination by negative-ion MALDI-QIT-TOFMS ${ }^{n}$ after pyrene derivatization of variously fucosylated oligosaccharides with branched decaose cores from human milk. Glycobiology 19, 601614 (2009)

34. Chai, W., Lawson, A.M., Piskarev, V.: Branching pattern and sequence analysis of underivatized oligosaccharides by combined MS/MS of singly and doubly charged molecular ions in negative-ion electrospray mass spectrometry. J. Am. Soc. Mass Spectrom. 13, 670-679 (2002)

35. Chai, W., Piskarev, V., Lawson, A.M.: Negative-ion electrospray mass spectrometry of neutral underivatized oligosaccharides. Anal. Chem. 73, 651-657 (2001)

36. Pfenninger, A., Karas, M., Finke, B., Stahl, B.: Structural analysis of underivatized neutral human milk oligosaccharides in the negative ion mode by nano-electrospray $\mathrm{MS}^{n}$, Part 1: methodology. J. Am. Soc. Mass Spectrom. 13, 1331-1340 (2002)

37. Pfenninger, A., Karas, M., Finke, B., Stahl, B.: Structural analysis of underivatized neutral human milk oligosaccharides in the negative ion mode by nano-electrospray $\mathrm{MS}^{n}$, PART 2: application to isomeric mixtures. J. Am. Soc. Mass Spectrom. 13, 1341-1348 (2002) 